\title{
Desgaste de dressadores de ponta única fabricados com diamantes cvd nacionais
}

\section{(Single point wear dressers manufactured with national cvd diamonds)}

\author{
A. Frech Jr., E. C. Bianchi, R. C. Canarim, P. R. Aguiar \\ Faculdade de Engenharia de Bauru - FEB, UNESP, Av. Eng. Luiz Edmundo C. Coube 14-01, \\ Bauru, SP 17033-360 \\ arfrech@gmail.com,bianchi@feb.unesp.br rubenscanarim@hotmail.com,aguiarpr@feb.unesp.br
}

\begin{abstract}
Resumo
Neste trabalho, buscou-se observar a atuação de três tipos de diamantes, dois naturais e um sintético, para determinar qual deles apresenta vida útil maior ou com maior desgaste em relação aos demais. A metodologia empregada nesse estudo foi o de dressar um rebolo de óxido de alumínio e, a cada vinte passadas com um avanço da profundidade de dressagem de $40 \mu \mathrm{m}$ por passada, tirar uma imagem da ponta na posição horizontal e outra na posição vertical deste diamante num microscópio estereoscópio. Após isso, o dressador foi recolocado na retificadora para a realização de uma nova dressagem. O critério para o fim do ensaio foi o faiscamento, gerado pelo contato do rebolo com a base do dressador, que indicou o fim do contato do diamante com o rebolo. Após os ensaios, foram calculadas as áreas gastas de cada diamante de acordo com cada imagem obtida por meio do auxilio de um software gráfico. Os dados foram então dispostos em gráficos.

Palavras-chave: dressagem, retificação,desgaste do dressador.
\end{abstract}

\begin{abstract}
This study attempted to observe the performance of three types of diamonds, two natural and one synthetic, to determine which features longer life and greater wear than the others. The methodology employed in this study was to dressed an aluminum oxide grinding wheel, and every twenty paces with a advance of depth dressing of $40 \mu \mathrm{m}$ per passes, take an image of the tip in a horizontal position and the other in the vertical position of this diamond in a stereoscopic. Thereafter, the dresser was replaced in the rectifier to conduct a new dressing. The criteria to completion of the test was the spark generated by the grinding wheel contact with the base of the dresser, which indicates the end of the contact with the diamond wheel. After the tests, was calculated the areas worn of each diamond in accordance with each image obtained through the aid of a graphics software. The data were then arranged in graphs.

Keywords: dressing, grinding, wear dresser.
\end{abstract}

\section{INTRODUÇÃO}

A retificação é um processo de usinagem que utiliza um rebolo abrasivo rotacionando em alta velocidade para a remoção de volume de um material menos duro. Esta é uma tecnologia chave para a produção de produtos e superfícies avançadas em uma ampla gama de indústria [1]. O processo de retificação é principalmente utilizado para a confecção de peças com alta qualidade, para garantir alta exatidão e com mínimas tolerâncias. É principalmente aplicada para garantir alta precisão e qualidade [2]. Sendo uma das últimas etapas do processo de usinagem, a retificação necessita de uma grande precisão para um acabamento com um mínimo valor de defeitos possível. O comportamento de qualquer processo de retificação é muito dependente do desempenho da ferramenta. Esse desempenho pode mudar de maneira significativa durante o processo de retificação e isso dificulta a previsão a respeito do comportamento do processo durante seu andamento. [2]. O rebolo, além de sofrer alterações durante o processo de retificação, pode perder suas condições ideais em seu próprio processo de fabricação. Assim, os resultados da retificação estão diretamente relacionados com as condições topográficas da superfície de trabalho do rebolo. Estando essas condições alteradas, a operação de dressagem passa a ser fundamental ao processo.

Dressagem é o processo de condicionamento da superfície do rebolo visando sua remodelação quando o mesmo perdeu sua forma original pelo desgaste [3]. É a operação conjunta de perfilamento e afiação dos rebolos convencionais [1]. As condições de dressagem podem provocar uma grande influência no desempenho da operação de retificação. Para se ter uma ideia dessa influência, basta dizer que as forças de retificação podem variar cerca de $500 \%$, apenas variando-se as condições de dressagem em um mesmo tipo de operação [4]. A ferramenta utilizada para a recuperação do rebolo é o dressador. Devido ao grande número de variáveis que influenciam o processo, existe um número muito grande de ferramentas (dressadores) que já foram desenvolvidas, e cada uma dessas ferramentas é responsável pela abrangência de um número limitado de casos. Dressadores de ponta única 
são utilizados para perfilar e dressar rebolos para operações de precisão, tais como retificação plana, cilíndrica, centerless e interna. Pontas simples de diamante, quando usadas em rebolos duros, largos, de grande diâmetro, podem ficar com áreas achatadas, as quais cegam o rebolo nas operações subsequentes de dressagem. $\mathrm{O}$ monitoramento deste processo se torna, então, necessário para o aprimoramento e o maior conhecimento a respeito das técnicas que devem ser utilizadas para a obtenção de um melhor rendimento.

A exploração de diamantes tem uma série de implicações no meio ambiente e desenvolvimento sócio econômico para a região onde ocorrem. A atividade da indústria diamantífera pode trazer como resultado uma progressiva degradação dos solos, diminuindo a biodiversidade, poluição das águas dos rios, desmatamento e desflorestamento de vastas áreas. Além desses problemas que podem ser gerados ainda existem os ligados aos resíduos sólidos que não encontram um destino e um tratamento satisfatório. Num ambiente maior, a extração de diamantes cria erosão dos solos, poluição do ar, desvia o curso de rios e altera as propriedades organoléticas da água. A mineração de diamante influencia também no ambiente social e econômico da região, trazendo desenvolvimento econômico, mas também gerando forte dependência da comunidade nessa atividade. Dessa forma, durante uma crise econômica, por exemplo, a exploração do diamante sofre uma queda em sua produção, gerando demissões nessa área de mineração.

Muitas são as medidas para diminuir os estragos ambientais na mineração, como leis e medidas de fiscalização e emprego de tecnologias que agridam cada vez menos ao meio ambiente. Nesse ponto, o uso de diamantes sintéticos pode ser uma saída para a diminuição da exploração de diamantes pelo mundo. $\mathrm{O}$ diamante usado em joias e artigos de luxo ainda seria explorado com a extração direta da natureza, porém os diamantes de uso industrial não teriam mais essa dependência da exploração diamantífera. Assim, são importantes as pesquisas científicas voltadas para a área dos diamantes sintéticos, que num futuro cada vez mais próximo possam tomar o lugar dos diamantes naturais para uso industrial, reduzindo o uso de minérios advindos de áreas de extração e exploração de diamantes.

Sendo o conhecimento a respeito da operação de dressagem pouco explorado, com bibliografias limitadas, este ainda é superficial. Para aprofundar este conhecimento acerca de um tipo específico de dressador, dentre os vários existentes, propõe-se neste trabalho a realização de ensaios monitorados com dressadores de ponta única, sendo o principal material de estudo o diamante CVD nacional. A técnica de fabricação de diamantes sintéticos em altas pressões já era dominada por várias empresas nas décadas de 50 e 60, essas pressões eram de acima de $60.000 \mathrm{~atm}$ e temperaturas acima de $2000 \mathrm{~K}$ [5]. As aplicações principais para esse diamante sintético eram em ferramentas de corte e para polimento de superfícies. Na década de 70 que começaram a surgir trabalhos de pesquisa acadêmicos que buscavam obter pequenas camadas de diamantes a partir de gases que possuíam carbono em sua composição [6]. Essas camadas, chamadas de filmes, eram produzidas em pressões menores do que a uma atmosfera e em temperaturas abaixo de $1200 \mathrm{~K}$, em substratos incrustados com pó de diamante. A partir disso, a técnica de obtenção de diamante a partir de um gás precursor se disseminou pelo mundo, como um método muito mais fácil e produtivo do que o utilizado nas décadas de 50 e 60. Esse diamante sintético advindo desta técnica passou-se a chamar diamante CVD [7].

O grande interesse pela síntese do diamante é de suas propriedades físico-químicas, onde o diamante é o material mais duro conhecido, 10 pela escala Mohs, possui baixa densidade, $3,5 \mathrm{~g} / \mathrm{cm}^{3}$, baixo coeficiente de expansão térmica, $0,8 \times 10^{-6} /{ }^{\circ} \mathrm{C}$ a $293 \mathrm{~K}$, alto índice de refração, 2,42, condutividade térmica maior do que qualquer outro material à temperatura ambiente, $20 \mathrm{~W} / \mathrm{cm}-{ }^{\circ} \mathrm{C}$, transparência óptica que vai desde a região do infravermelho à da luz visível, é um excelente isolante elétrico, inerte quimicamente, apresenta compatibilidade biológica e quando dopado tornase um bom semicondutor [7].

Existem vário métodos de obtenção do diamante CVD que diferem basicamente pelo processo utilizado na decomposição e ativação da mistura gasosa, cujo qual resultará num gás ionizado, conhecido como plasma. As técnicas conhecidas são o plasma gerado por micro-ondas - MWCVD - [8], filamento quente - HFCVD - [9], chama de acetileno e oxigênio - ATCVD - [10], jato de plasma - AJCVD - [11]. Apesar do grande número de técnicas utilizadas para a obtenção de filmes com boa qualidade, esses processos continuam em fase de contínuos testes, onde uma vasta quantidade de trabalhos está sendo publicado [12]. As principais dificuldades que são encontradas na produção e aplicação do diamante CVD são na influência das propriedades químicas do substrato, no aumento da taxa de nucleação e na adesão do filme [6]. Ademais, nenhuma das películas que já foram produzidas possui a regularidade cristalina em larga escala dos diamantes naturais. As propriedades mecânicas, elétricas e óticas das películas de diamante já sintetizados se aproximam daquelas do diamante bruto, mas ainda não de forma altamente satisfatória [13].

As propriedades mecânicas do diamante natural têm sido extensivamente pesquisadas nas últimas cinco décadas, mas mesmo considerando as várias pesquisas feitas sobre o tema, poucas alcançaram alto nível de satisfação, devido às características impar do diamante. Como exemplo, na falta de entendimento na área do entendimento dos termos básicos da deformação mecânica do diamante, num modelo de explicar a anisotropia de friç̧ão e num mecanismo eficiente de polimento da superfície do diamante [6].

$\mathrm{O}$ diamante natural pode ser definido, em temperaturas e pressões atmosféricas ambientais, como um polimorfo metaestável do carbono. As propriedades físicas do diamante natural o tornam extremamente atrativo. Ele é extremamente duro - o material duro mais conhecido - e possui uma condutividade elétrica muito baixa. Essas características são devidas à sua estrutura cristalina e às fortes ligações interatômicas covalentes. O diamante natural apresenta ainda uma condutividade térmica alta para um material não- 
metálico, além de ser oticamente transparente nas regiões visível e infravermelho do espectro eletromagnético, e possuir um elevado índice de refração [13].

Monocristais de diamante relativamente grandes e com alto índice de pureza são usados como pedras preciosas para joias. Industrialmente, os diamantes são utilizados para trituras ou cortar outros materiais mais moles. A grande desvantagem do diamante natural para o uso industrial é seu elevado custo e, atualmente, na dificuldade de encontrá-los na natureza [13].

\section{EXPERIMENTAL}

Para a realização dos ensaios foram utilizados nove dressadores de ponta única de três tipos diferentes, uma retificadora plana fabricada pela Sulmecânica, modelo 1055E e um microscópio estereoscópio marca Carl Zeiss. Para a realização dos ensaios, foram utilizados também três rebolos fabricados pela empresa Norton, feitos de abrasivo convencional de óxido de alumínio, com ligante vitrificado, dureza L - média -, granulometria 150 - muito fina - e estrutura com dimensões $355,6 \mathrm{~mm}$ x $25,4 \mathrm{~mm}$ x $127 \mathrm{~mm}$. Dentre os principais parâmetros de retificação e dressagem utilizados, tem-se que a velocidade transversal do rebolo foi mantida constante para os ensaios dos três tipos de dressadores. A velocidade do inversor do motor da retificadora também foi constante para os ensaios, bem como a frequência. Assim, tem-se a velocidade transversal igual a $3,45 \mathrm{~mm} / \mathrm{s}$, a velocidade do inversor igual a 450 rpm e a frequência de $60 \mathrm{~Hz}$. A profundidade de dressagem (ad) foi constante e igual a $40 \mu \mathrm{m}$. O parâmetro de dressagem do grau de recobrimento (Ud) foi de 1 (um). Todas as dressagens foram feitas sem o uso de fluido de corte, ou seja, a seco. Os ensaios consistiam em colocar um dressador de ponta única, tal como o mostrado na Fig. 1, de um dos três tipos de diamantes (CVD Sintético ou Brasil Extra ou Mato Grosso) em contato com o rebolo e realizar vinte passadas. Após as passadas, o dressador tinha sua ponta do diamante fotografada por meio do microscópio estereoscópio. O dressador então retornava à máquina para mais vinte passadas.

Quando se verificava no processo de dressagem uma forte

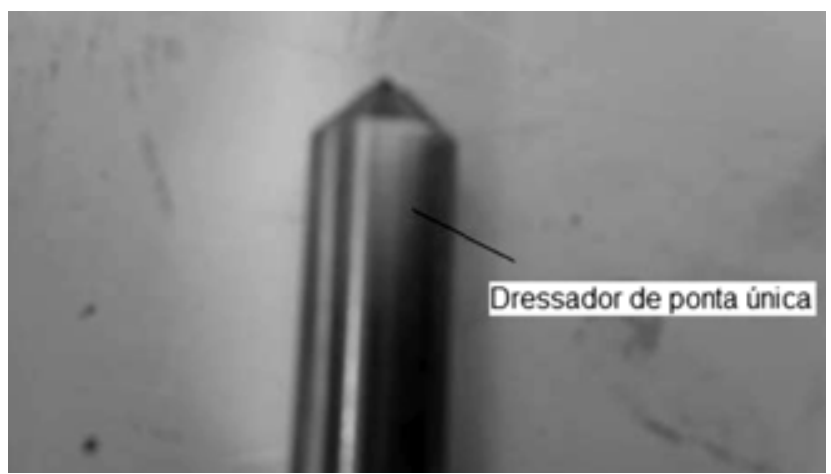

Figura 1: Dressador de ponta única com ponta de diamante Royall Brasil Extra.

[Figure 1: Single point dresser with diamond type Brasil Extra.]

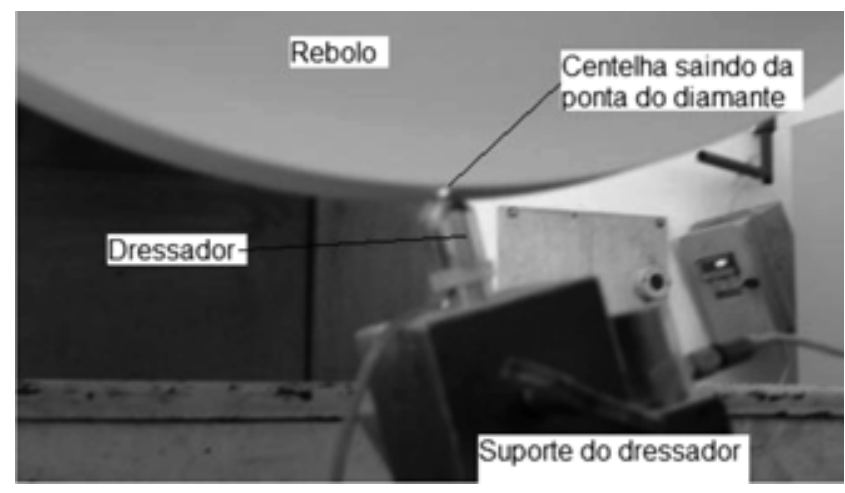

Figura 2: Fim de vida do diamante, mostrando o faiscamento da base metálica do dressador com o rebolo.

[Figure 2: End of diamond life, showing the spark of the base metal of the dresser with the wheel.]

incidência de fagulhas saindo do dressador era determinado o fim do ensaio, mostrando que a base metálica do dressador estava entrando em contato com o rebolo. Foram realizados no total três ensaios completos para cada tipo de dressador e utilizando três rebolos, dando um total de nove ensaios. O esquema de montagem do sistema e a caracterização do fim do ensaio está sendo mostrada pela Fig. 2.

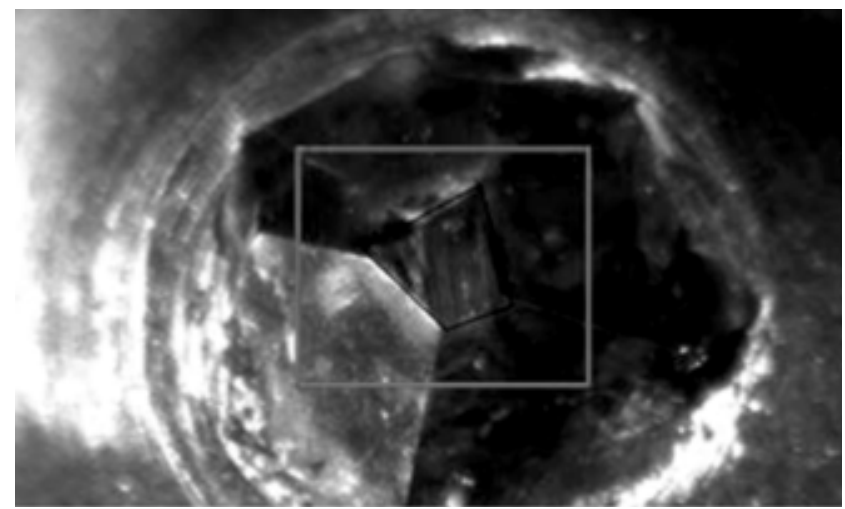

Figura 3: Esquema para encontrar a área por meio do software. [Figure 3: Scheme to find the area through the software.]

As fotografias dos diamantes eram retiradas na posição horizontal e vertical do dressador. Com a utilização do software gráfico Solid Edge ST2 foi possível calcular aproximadamente a área desgastada do diamante pelo processo de dressagem para as duas posições. A forma de medição da área é mostrada na Fig. 3.

\section{RESULTADOS E DISCUSSÃO}

\section{Diamante CVD Sintético}

Para o dressador de ponta única com o diamante tipo CVD Sintético Nacional foram realizados três ensaios. Os valores médios encontrados para a área desgastada na posição horizontal a cada vinte passadas a partir dos três ensaios são mostradas na Fig. 4, juntamente com seus respectivos desvios padrões. 
O primeiro ensaio deste diamante teve uma durabilidade de 156 passadas, contra 295 passadas do segundo ensaio e 184 passados totais para o terceiro ensaio. Para melhores valores estatísticos e comparação, os dados acima de 140 passadas foram descartados.

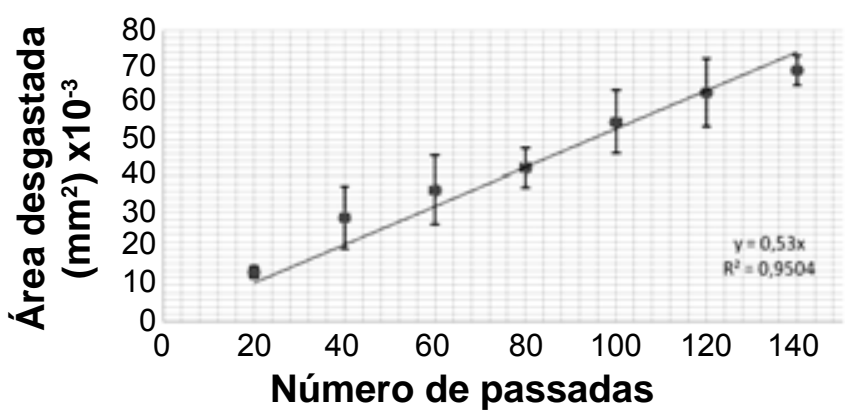

Figura 4: Área desgastada versus número de passadas para o diamante CVD sintético (horizontal).

[Figure 4: Worn area versus number of paces for CVD synthetic Diamond (horizontal).]

Pela Fig. 4 observa-se uma linearidade entre o dado pelo ajuste linear obtido pela curva do gráfico, sendo de 0,9504 . O desvio padrão encontrado e exposto indica média dispersão dos dados. O coeficiente angular da reta encontrada na Fig. 4 é de 0,5286 área desgastada pelo número de passadas. Dessa forma, para cada passada são gastos $0,5286 \mathrm{~mm} 2 \mathrm{de}$ área.

As imagens das pontas desgastadas do diamante CVD

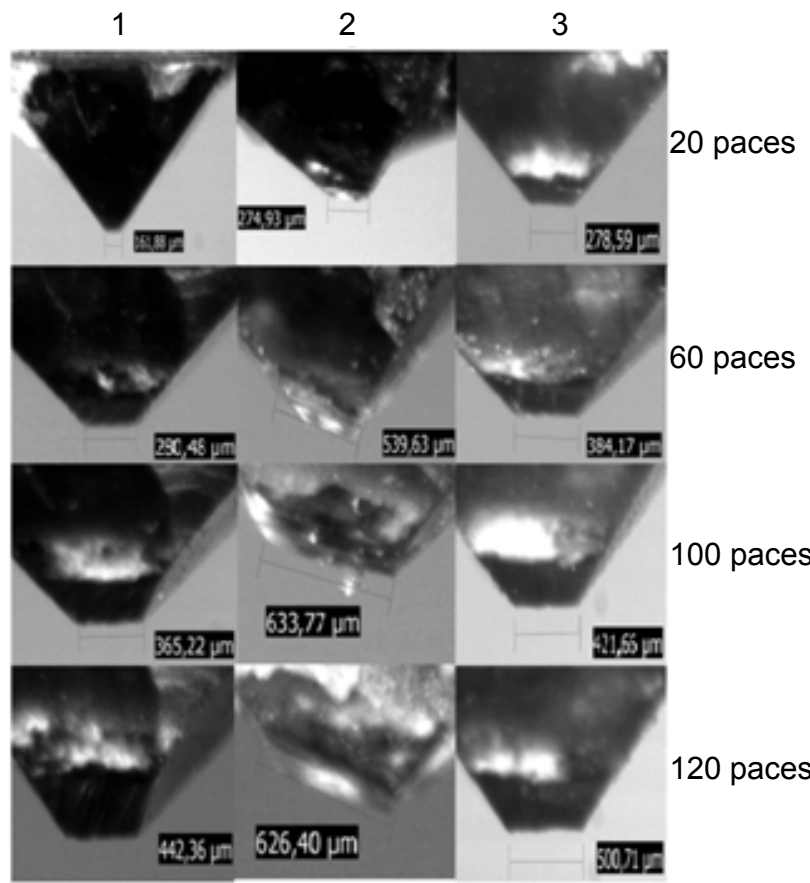

Figura 5: Imagens das pontas dos diamantes CVD Sintéticos na posição horizontal, onde as colunas representam os ensaios - 1, 2 e 3 - e as linhas o número de passadas - 20, 60, 100 e 120.

[Figure 5: Images of tips of CVD synthetic diamonds in the horizontal position, where the columns represent the tests - 1, 2 and 3 - and lines the number of paces - 20,60,100 and 120.]
Sintético Nacional na posição vertical estão na Fig. 5.

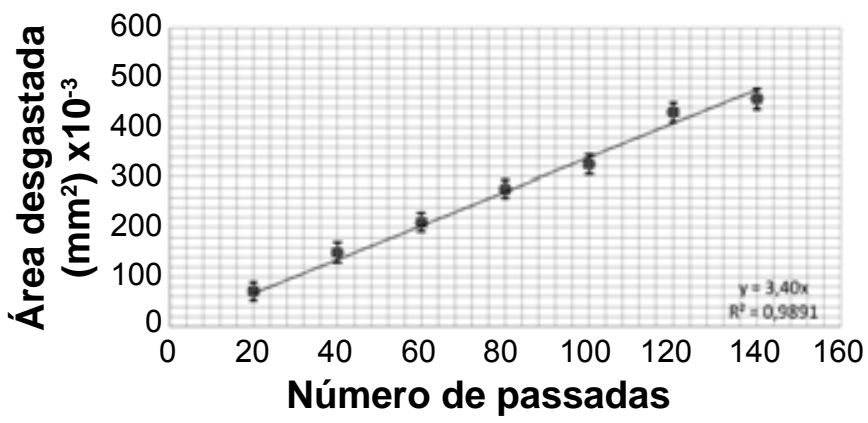

Figura 6: Área desgastada versus número de passadas para o diamante CVD sintético (vertical).

[Figure 6: Worn area versus number of paces for CVD synthetic diamond (vertical).]

Para a posição vertical, os dados obtidos estão dispostos na Fig. 6, com os valores de área médias dos três ensaios com seus respectivos desvios-padrões

Observando a curva da Fig. 6 observa-se que os dados de área desgastada apresentaram uma boa disposição linear, bastando verificar o ajuste linear da curva obtida com 0,9891 , extremamente próximo de 1 . O desvio padrão obtido também indica baixa dispersão dos dados. O coeficiente angular da reta encontrada na Fig. 6 é de uma taxa de desgaste de $3,3968 \mathrm{~mm}^{2}$ de área desgastada por número de passadas. Dessa forma, para uma passada são gastos $3,3968 \mathrm{~mm}^{2}$ de área.

As imagens referentes às pontas dos diamantes CVD sintético para a posição vertical são mostradas na Fig. 7.

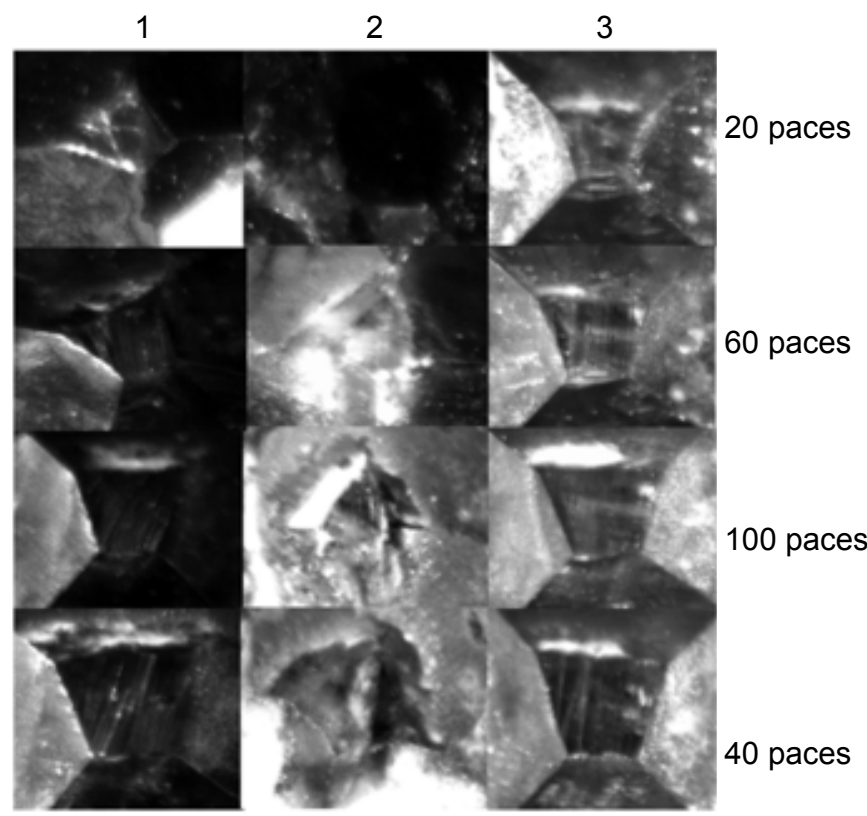

Figura 7: Imagens das pontas dos diamantes CVD sintéticos na posição vertical, onde as colunas representam os ensaios - 1, 2 e 3 - e as linhas o número de passadas - 20,60, 100 e 120.

[Figure 7: Images of tips of CVD synthetic diamonds in the vertical position, where the columns represent the tests - 1, 2 and 3 - and lines the number of paces - 20,60,100 and 120.] 


\section{Diamante Brasil Extra}

Para o dressador de ponta única com o diamante tipo Brasil Extra foram realizados três ensaios. Os valores médios encontrados para a área desgastada a cada vinte passadas na posição horizontal a partir dos três ensaios estão dispostas na Fig. 8, juntamente com seus respectivos desvios padrões. $\mathrm{O}$ primeiro ensaio deste diamante apresentou 180 passadas. O segundo ensaio atingiu 166 passadas e o terceiro, 117 passadas totais. Para melhores efeitos comparativos, os dados acima de 100 passadas

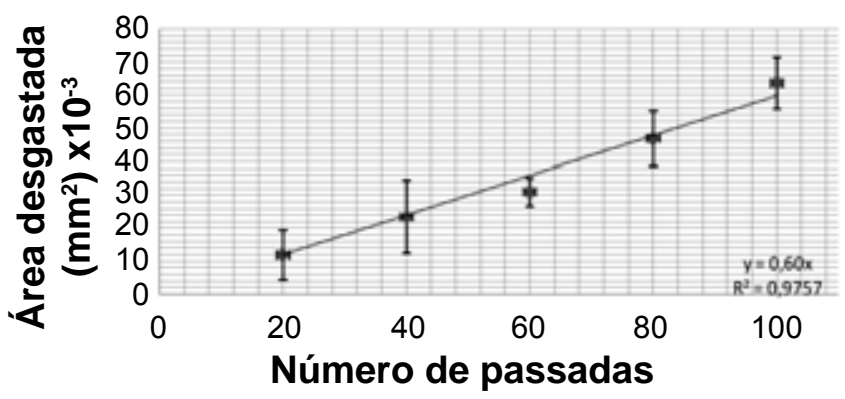

Figura 8: Área desgastada versus número de passadas para o diamante Brasil Extra (horizontal).

[Figure 8: Worn area versus number of paces for the Brazil diamond Extra (horizontal).]

foram descartados.

Pela Fig. 8 observa-se uma linearidade entre os dados, com um ajuste linear da curva mostrada com 0,9757. O desvio padrão também está indicando uma média dispersão dos dados. O coeficiente angular da reta encontrada é de $0,5972 \mathrm{~mm}^{2}$ de área desgastada por número de passadas. Dessa forma, para cada passada são gastos $0,5972 \mathrm{~mm}^{2} \mathrm{de}$ área.

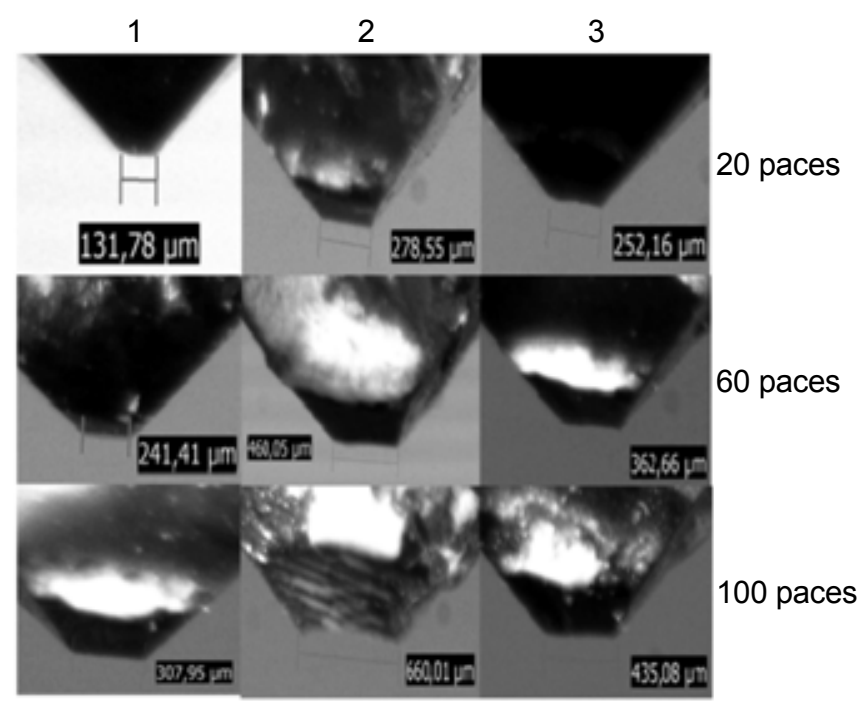

Figura 9: Imagens das pontas dos diamantes Brasil Extra na posição horizontal, onde as colunas representam os ensaios - 1, 2 e 3 - e as linhas o número de passadas - 20, 60 e 100.

[Figure 9: Images from the tips of diamonds Brazil Extra in horizontal position, where the columns represent the tests - 1, 2 and 3 - and the number of rows in the paces - 20, 60 and 100.]
As imagens referentes às pontas dos diamantes Brasil Extra para a posição horizontal são mostradas na Fig. 9.

Para a posição vertical, foram obtidos os dados apresentados na Fig. 10, com a média da área desgastada e seus respectivos desvios-padrões.

Pelos dados obtidos e mostrado pela Fig. 10, observa-

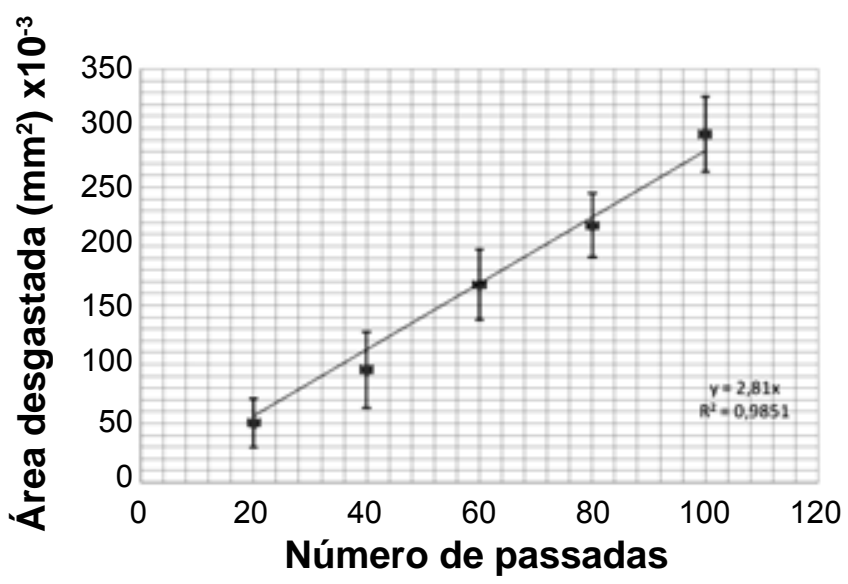

Figura 10: Área desgastada versus número de passadas para o diamante Brasil Extra (vertical).

[Figure 10: Worn area versus number of paces for the Brazil diamond Extra (vertical).]

se uma linearidade entre os dados, como mostrado no ajuste linear obtido de 0,9851 . O desvio padrão indica baixa dispersão dos dados. O coeficiente angular da reta encontrada na Fig. 10 é de $2,8067 \mathrm{~mm}^{2}$ de área desgastada. Dessa forma, para uma passada são gastos $2,8067 \mathrm{~mm}^{2} \mathrm{de}$ área.

1

2

3

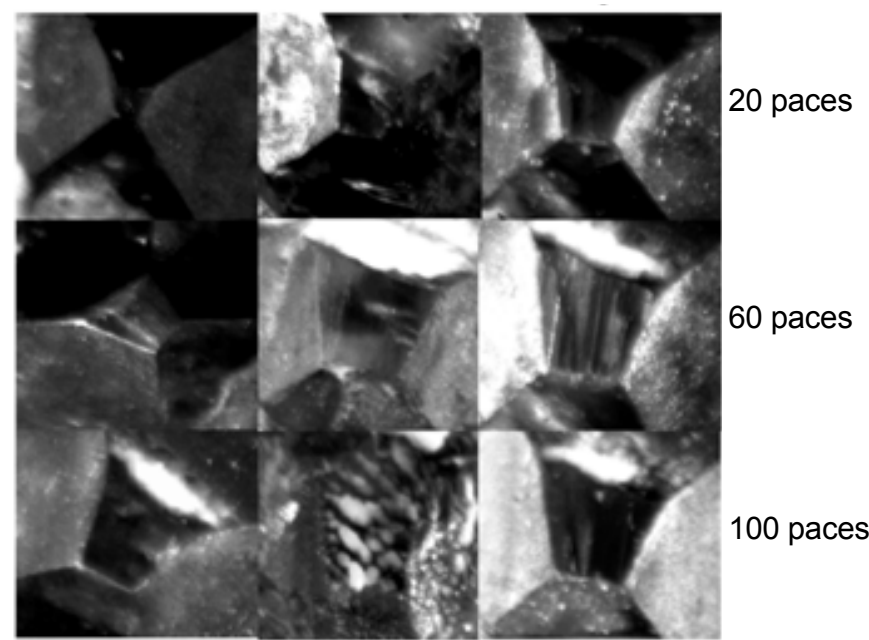

Figura 11: Imagens das pontas dos diamantes Brasil Extra na posição vertical, onde as colunas representam os ensaios - 1, 2 e 3 - e as linhas o número de passadas - 20,60 e 100.

[Figure 11: Images from the tips of diamonds Brazil Extra in vertical position, where the columns represent the tests - 1, 2 and 3 - and the number of rows in the paces - 20, 60 and 100.]

As imagens referentes às pontas dos diamantes Brasil Extra para a posição horizontal são mostradas na Fig. 11. 


\section{Diamante Mato Grosso}

Para o dressador de ponta única com o diamante tipo Mato Grosso foram realizados três ensaios. Os valores médios encontrados para a área desgastada para a posição horizontal a cada vinte passadas a partir dos três ensaios estão dispostas na Fig. 12, juntamente com seus respectivos desvios padrões. O primeiro ensaio obteve 140 passadas totais até o final do ensaio. Para o segundo ensaio o total de passadas foram de 191 passadas. Já para o terceiro ensaio foram 86 passadas. Para melhores comparações, os dados acima de 100 passadas foram descartados.

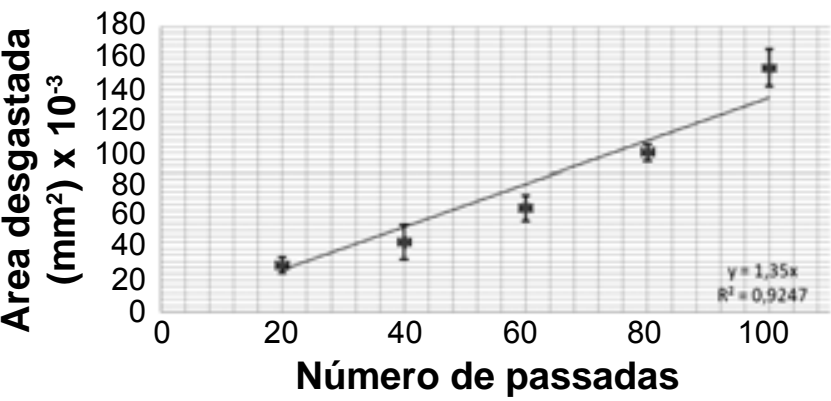

Figura 12: Área desgastada versus número de passadas para o diamante tipo Mato Grosso (horizontal).

[Figure 12: Worn area versus number of paces for the diamond kind Mato Grosso (horizontal).]

Pela Fig. 12 observa-se uma linearidade entre os dados, visto que o ajuste linear obtido para a reta ser de 0,9247 . O desvio padrão encontrado também está indicando média dispersão dos dados. O coeficiente angular da reta encontrada é de $1,3514 \mathrm{~mm}^{2}$ de área desgastada por número de passadas. Dessa forma, para uma passada são gastas

1
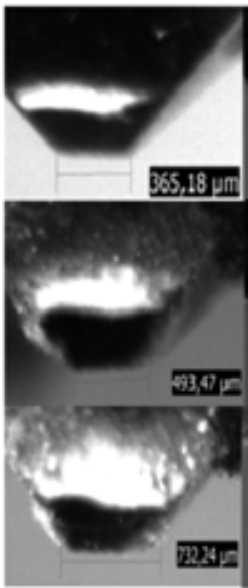

Figura 13: Imagens das pontas dos diamantes Mato Grosso na posição horizontal, onde as colunas representam os ensaios - 1, 2 e 3 - e as linhas o número de passadas - 20,60 e 100.

[Figure 13: Images from the tips of diamonds Mato Grosso in the horizontal position, where the columns represent the tests - 1, 2 and 3 - and the number of rows in the paces - 20, 60 and 100.]

\section{$1,3514 \mathrm{~mm}^{2}$ de área.}

As imagens referentes às pontas dos diamantes Brasil Extra para a posição horizontal são mostradas na Fig. 13.

Os dados obtidos para a posição vertical estão apresentados na Fig. 14, com suas médias e respectivos desvios-padrões.

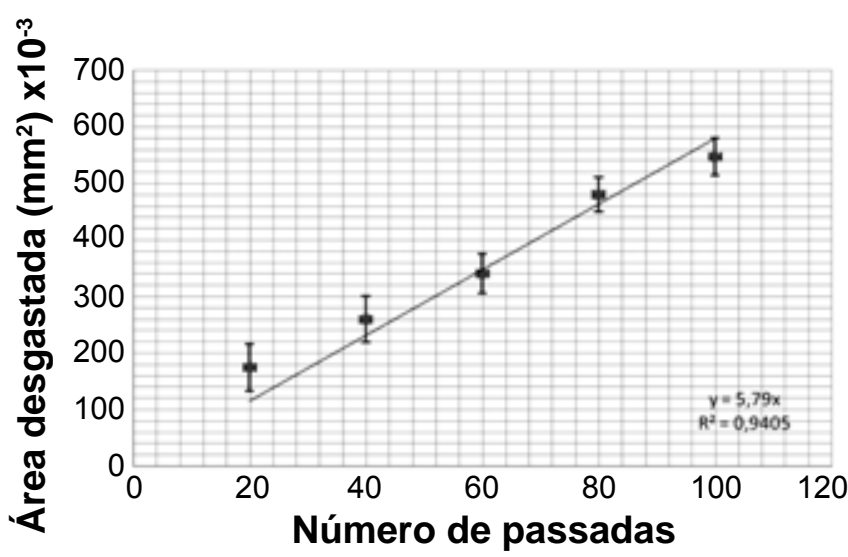

Figura 14: Área desgastada versus número de passadas para o diamante tipo Mato Grosso (vertical).

[Figure 14: Worn area versus number of paces for the diamond kind Mato Grosso (vertical).]

Pela Fig. 14 observa-se uma linearidade entre os dados, pelo ajuste linear da reta de 0,9405 . O desvio padrão indica relativa baixa dispersão dos dados. O coeficiente angular da reta ajustada na Fig. 14 é $5,7932 \mathrm{~mm}^{2}$ de área desgastada por número de passadas. Dessa forma, para cada passada são gastos $5,7932 \mathrm{~mm}^{2}$ de área.

As imagens referentes às pontas dos diamantes Brasil Extra para a posição horizontal são mostradas na Fig. 15.

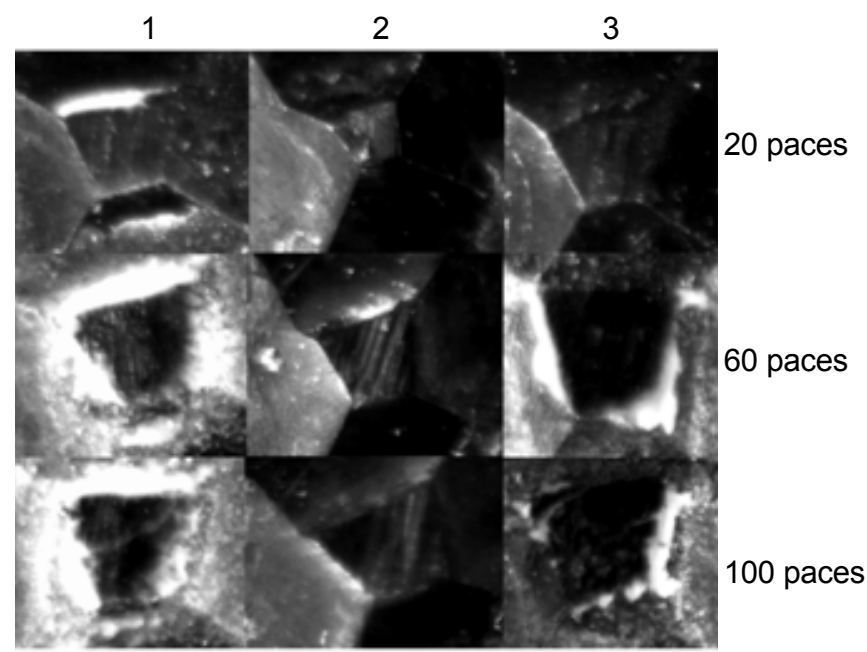

Figura 15: Imagens das pontas dos diamantes Mato Grosso na posição vertical, onde as colunas representam os ensaios - 1,2 e 3 - e as linhas o número de passadas - 20, 60 e 100.

[Figure 15: Images from the tips of diamonds Mato Grosso in the vertical position, where the columns represent the tests - 1, 2 and 3 - and the number of rows in the paces - 20, 60 and 100.] 


\section{Comparações entre os três diamantes}

\section{Horizontal}

Pelas Figs. 4, 8 e 12 observa-se que o dressador do tipo Mato Grosso foi o que teve a maior relação área desgastada pelo número de passadas. Os diamantes do tipo CVD sintético e Brasil Extra se comportaram de forma muito parecida, com o CVD sintético num desgaste maior que o Brasil Extra até próximo a passada 80, quando o Brasil Extra apresenta uma maior área desgastada. Observando também pelos coeficientes angulares dos três tipos de diamantes obtidos pelas Figs. 4, 6 e 8, o diamante tipo Mato Grosso apresenta um coeficiente de $1,35 \mathrm{~mm}^{2} /$ passada, contra $0,60 \mathrm{~mm}^{2} /$ passada do Brasil Extra e $0,53 \mathrm{~mm}^{2} /$ passada do CVD sintético, mostrando claramente que o diamante Mato Grosso apresenta um maior desgaste com relação aos outros dois. Portanto, o dressador do tipo Mato Grosso apresenta um maior desgaste da área do diamante que o do diamante sintético e o Brasil Extra, numa relação de $39,11 \%$ a mais de desgaste para o diamante Sintético e $43,82 \%$ a mais de desgaste para o diamante Brasil Extra. Assim, o diamante tipo Mato Grosso é de qualidade inferior. Para os diamantes tipo CVD sintético e Brasil Extra, as relações de desgaste da área foram muito próximas, com uma ligeira superioridade do diamante CVD sintético em longo prazo, sendo que este apresentou também maior tempo de vida que os demais. Assim, a relação de desgaste por passada do Brasil Extra para o CVD Sintético nacional é de apenas 10,76\% a mais de desgaste do Brasil Extra para o CVD Sintético.

\section{Vertical}

Nas Figs. 6, 10 e 14 observa-se que o dressador possuidor do diamante tipo Mato Grosso foi o que teve maior relação área desgasta por número de passadas que os demais. O diamante tipo CVD Sintético apresentou levemente uma maior relação maior área desgastada por número de passadas que o diamante tipo Brasil Extra. Com os dados dos coeficientes angulares obtidos pelas Figs. 6, 10 e 14, o diamante tipo Mato Grosso apresenta $5,79 \mathrm{~mm}^{2} /$ passada, enquanto que o CVD sintético apresenta $3,40 \mathrm{~mm}^{2} /$ passada e $2,81 \mathrm{~mm}^{2} /$ passada. Dessa forma, o diamante Mato Grosso apresentou uma relação de $58,63 \%$ a mais de desgaste que o diamante CVD sintético e 48,45\% a mais de desgaste que o diamante Brasil Extra. O diamante CVD sintético nacional apresentou maior taxa de área gasta por passada que o Brasil Extra, com uma relação de $17,37 \%$ a mais de desgaste. Da mesma forma que para os dados obtidos para a posição horizontal, o diamante Brasil Extra apresentou relação de $10,76 \%$ a mais de desgaste que o diamante sintético. Assim, pode-se verificar uma aproximação nos resultados, mostrando que tanto o diamante CVD sintético Nacional quanto o Brasil Extra apresentam resultados próximos. Para o diamante Mato Grosso em relação ao CVD sintético obteve-se uma relação de $39,11 \%$ a mais de desgaste para a posição horizontal e $58,63 \%$ a mais de desgaste para posição vertical. Para a relação do diamante Mato Grosso em relação ao Brasil Extra tem-se $43,82 \%$ a mais de desgaste para a posição horizontal e $48,45 \%$ a mais de desgaste para a posição vertical. Assim, os dados apresentam bastante proximidade, confirmando que tanto a posição horizontal quanto a posição vertical apresentam forte correspondência.

\section{CONCLUSÕES}

Com os dados obtidos para os três tipos diferentes de diamantes que o dressador com a ponta de diamante tipo Mato Grosso apresentou uma maior relação de área desgasta do diamante pelo número de passadas que os dressadores de diamante do tipo CVD Sintético Nacional e tipo Brasil Extra. O diamante tipo Mato Grosso mostrou também a maior discrepância em relação ao número de passadas por ensaio. Dessa forma, é possível concluir que o diamante tipo Mato Grosso é então, com base única e exclusivamente nos parâmetros utilizados e nos ensaios realizados para a confecção deste trabalho, o de menor qualidade entre todos os três diamantes testados. O dressador com ponta de diamante tipo Brasil Extra apresentou uma relação área desgastada pelo número de passadas muito próxima da do diamante CVD sintético. Porém, o diamante Brasil Extra apresentou o menor tempo de vida dentre os três diamantes, com um número de passadas máxima de 180 - conseguidas no primeiro ensaio -, contra 191 passadas obtidas pelo diamante tipo Mato Grosso no segundo ensaio e 295 passadas do diamante tipo CVD Sintético obtidos no segundo ensaio para esse diamante. $\mathrm{O}$ dressador com ponta de diamante tipo CVD sintético Nacional apresentou boa taxa de desgaste por passada, também muito próxima da obtida pelo diamante tipo Brasil Extra. Porém, o grande diferencial do diamante sintético é sua durabilidade, conseguindo um grande número de passadas quando comparada aos outros dois diamantes. Sendo o rebolo do mesmo tipo e os parâmetros mantidos constantes, a variável em questão era a durabilidade de cada diamante. $\mathrm{O}$ fenômeno envolvido para a durabilidade do diamante, ou seja, que explicam a diferença de desgaste e vida útil é a dureza. A dureza tende a diminuir à medida que a temperatura aumenta, dessa forma, os diamantes que mais absorveram temperatura tenderam a diminuir sua dureza. O diamante tipo Mato Grosso apresentou, numa situação de trabalho prolongado, ser menos resistente quanto a sua dureza. O diamante tipo Brasil Extra, apesar de bons resultados para área desgastada, não manteve uma boa dureza ao longo dos ensaios. Comparando com o diamante sintético, este obteve uma dureza constante, mesmo a altas temperaturas, mostrando uma vida útil alta. Portanto concluise que o diamante tipo Mato Grosso é de qualidade inferior, enquanto que os diamantes tipo CVD sintético Nacional e Brasil Extra são de maior qualidade, apresentando valores aproximados de taxa de desgaste, mas com uma grande vantagem do diamante tipo CVD sintético Nacional quando se refere ao tempo de vida do dressador, apresentando uma vantagem considerável, enquanto que o Brasil Extra foi o que apresentou menor vida útil. 


\section{AGRADECIMENTOS}

Os autores gostariam de agradecer à FAPESP (Fundação de Amparo à Pesquisa do Estado de S. Paulo) e ao IPEN (Instituto de Pesquisas Nucleares) pelo suporte dado a esta pesquisa e à UNESP - Universidade Estadual Paulista, onde esta pesquisa foi conduzida.

\section{REFERÊNCIAS}

[1] I. D. Marinescu, M. Hitchiner, E. Uhlmann, W. B. Rowe, I. Inasaki, "Handbook of machining with grinding wheels", $1{ }^{a}$ Ed., CRC Press, Taylor \& Francis Group, Boca Raton, FL, EUA (2007).

[2] R. X. Gao, "Monitoring Systems for Grinding Processes", Springer Series in Adv. Manufacturing (2007) 83-107.

[3] L. Xue, F. Naghdy, C. Cook, FIEEE Int. Conf. Ind. Techn., Bangkok, Tailândia (2002) 1296-1299.

[4] J. F. G. Oliveira, Análise da ação do macroefeito de dressamento de rebolos no desempenho do processo de retificação, Tese Dr., USP, S. Carlos, SP, Brasil (1998).

[5] R. F. Davis, Diamond films and coating: development, properties and applications, Noyes (1993) 421.

[6] C. A. Brookes, Diamond Related Mater. 1 (2010) 13-17.

[7] U. C. Angus, F. A. Buck, M. Sunkara, T. F. Groth, C. C. Hainan, MRS Bull. 10 (1995) 38.

[8] C. F. M. Borges, M. Moisan, A. Gicquel, Diamond Related Mater. 4 (1995) 149.

[9] S. Matsumoto, Y. Matsui, J. Mater. Sci. 18 (1983) 785.

[10] K. V. Ravi, C. A. Koch, H. S. Hn, J. Mater. Resistance 5 (1990).

[11] N. Ohtake, M. Yoshikawa, J. Electrochem. Soc. 137, 2 (1990) 717.

[12] V. J. Trava-Airoldi, E. J. Corat, L. V. Santos, A. V.Diniz, J. R. Moro, N. F. Leite, Diamond Related Mater. 11 (2002) 532.

[13] W. D. Callister, Estruturas e propriedades das cerâmicas, in "Ciência e engenharia de materiais: uma introdução", 5ª Ed., LTC, S. Paulo, SP, Brasil (2002) 275300.

(Rec. 12/10/2012, Rev. 07/06/2013, Ac. 08/06/2013) 